\title{
Performance Impairments in Single-Mode Radio-Over-Fiber Systems Due to MAC Constraints
}

\author{
B. Kalantari-Sabet, M. Mjeku, N. J. Gomes, Senior Member, IEEE, and J. E. Mitchell, Member, IEEE
}

\begin{abstract}
This paper investigates performance impairments due to constraints imposed by the MAC layer when single-mode fiber (SMF) is used to extend the reach of an IEEE 802.11 network. It is shown that data throughput decreases as fiber length increases. It is also noted that the network fails long before physical layer limitations set in due to the timeout values defined within the MAC protocol. This study is based on both variants of the IEEE 802.11 Distributed Coordination Function. Moreover, both UDP and TCP packet transmissions are taken into account. An experimental enquiry is initially developed to provide a set of validation points before extending these results by simulations using the OPNET platform. Finally, an analytical approximation is presented to these results that allows designers of Radio-over-fiber (RoF) systems to quickly and accurately predict the data throughput given the specific parameters of their network. To our knowledge, this is the first analysis of this kind for a long reach fiber system.
\end{abstract}

Index Terms-Broadband, IEEE 802.11, MAC, performance evaluation, Radio-over-fiber (RoF), TCP transmission, UDP transmission.

\section{INTRODUCTION}

$\mathbf{T}$ HE widespread integration of radio-over-fiber $(\mathrm{RoF})$ technology and wireless systems, specifically WiFi and WiMAX, provides a promising solution for both indoor and outdoor broadband access networks. RoF deploys optical fiber links, which have low loss and high bandwidth, to distribute radio frequency (RF) signals from a main unit called central site (CS) to remote antenna unit(s) (RAU). This technique can be used in the backbone of outdoor wireless networks such as the third-generation mobile or fixed wireless access (FWA) systems including WiFi and WiMAX. Fig. 1 demonstrates possible applications, showing both indoor and outdoor RoF systems where the main unit, i.e., the base transceiver station (BTS) or access point (AP), and its Antenna, i.e., the RAU, are separated and connected by means of single-mode fiber (SMF).

Manuscript received January 29, 2008; revised April 28, 2008. Current version published October 10, 2008. This work was supported in part by the U.K. Engineering and Physical Sciences Research Council (EPSRC) and by the FP6 EU Network of Excellence ISIS.

B. Kalantari-Sabet is with the Communications and Information Systems Group, Department of Electronic and Electrical Engineering, University College London, U.K. (e-mail: ksabet@ee.ucl.ac.uk).

M. Mjeku and N.J. Gomes are with the Broadband and Wireless Communications Group, Department of Electronics, University of Kent, Canterbury, U.K. (e-mail: ms246@kent.ac.uk; n.j.gomes@kent.ac.uk).

J. E. Mitchell is with the Communications and Information Systems Group, Department of Electronic and Electrical Engineering, University College London, U.K. (e-mail: j.mitchell@ee.ucl.ac.uk).

Color versions of one or more of the figures in this paper are available online at http://ieeexplore.ieee.org.

Digital Object Identifier 10.1109/JLT.2008.927158
Fig. 2 illustrates an analogous concept within the architecture of IEEE $802.11(\mathrm{a} / \mathrm{b} / \mathrm{g})$ standard.

The main advantage of RoF systems is the ability to concentrate most of the expensive, baseband processing and high frequency modulation equipment at CS, thereby making it possible to use compact and simpler RAUs. The functions carried out at the RAU are optical to electrical (O/E), electrical to optical (E/O) conversions and RF amplifications.

The feasibility of the above architecture has been demonstrated using multimode fiber (MMF) [1], [2]. It is shown that the fiber length could be in the range of $500 \mathrm{~m}$ to a few kilometers. In RoF systems, the longer the fiber length the higher the delay imposed to the system. For example, using $1 \mathrm{~km}$ of fiber introduces approximately $5 \mu$ s of delay to each packet that passes through. This extra propagation delay poses a challenge to the system design since this delay exceeds the $1 \mu$ s propagation delay boundary defined in the IEEE 802.11 standard where only wireless propagation is assumed [3]. Consequently, the performance of the distributed coordination function (DCF) of the medium access control (MAC) layer may degrade significantly or even break down entirely. Therefore, understanding the IEEE 802.11 MAC behavior in the presence of significant fiber length (delay) is of major importance.

A number of studies have investigated the effects of the fiber delay on the DCF of 802.11 MAC protocol [2], [4], [5]. However, none of them have presented accurate analytical approximations to predict the performance of such systems when specific parameters of the network are available. It is shown that the maximum fiber length that can be inserted in 802.11 WLAN is limited by the Acknowledgement (ACK) and Clear To Send (CTS) timeout parameters depending on the access mode used [2], [6].

This paper is organized as follows. Section II presents the IEEE 802.11 DCF mode within the context of hybrid opticalwireless systems. Then, by using an experimental enquiry a set of validation points are presented in Section III. In Section IV, we explain the simulation setup and introduce an analytical approximation that allows designers of RoF systems to quickly and accurately predict the data throughput given the specific parameters of their network. Section V presents and compares the results. A brief discussion on analytical approximation is covered in Section VI. Finally, Section VII concludes the paper.

\section{MAC PROTOCOL}

In the DCF mechanism, the IEEE 802.11 standard uses two methods to access the wireless medium. In the "Basic Access Method" or "two-way handshaking technique," after a successful transmission, the destination station waits for a short 


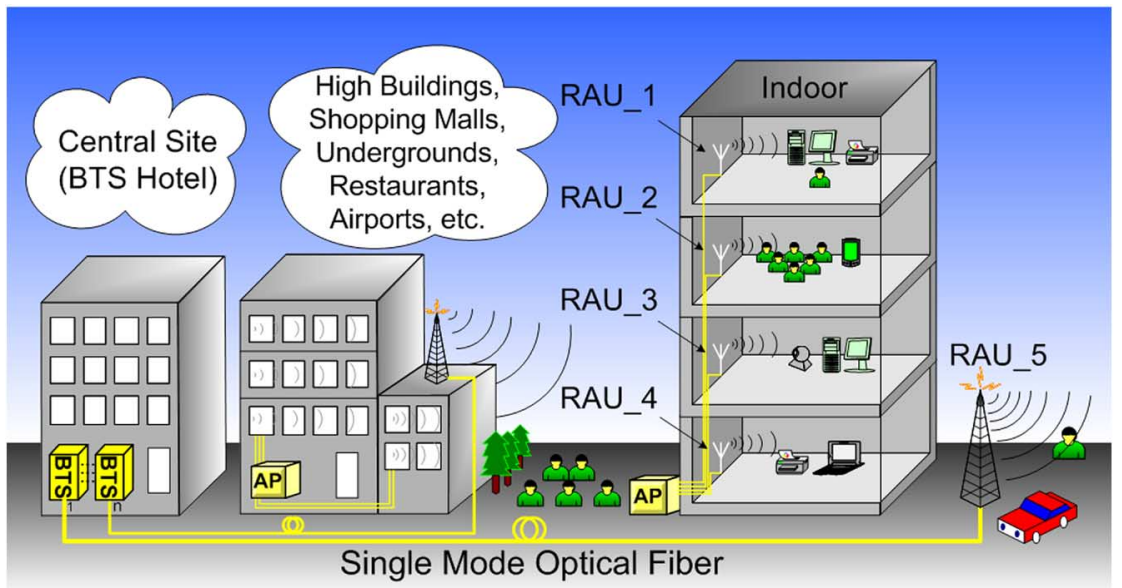

Fig. 1. Schematic of indoor and outdoor hybrid optical-wireless systems.

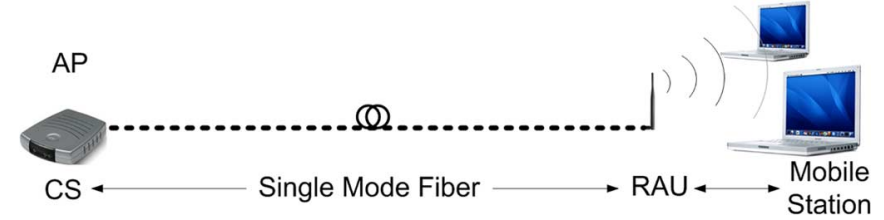

Fig. 2. Schematic of optically distributed $802.11(\mathrm{a} / \mathrm{b} / \mathrm{g})$ broadband network.

interframe space (SIFS) interval and then sends an ACK packet to confirm the correct reception of data at the MAC layer. After the transmission of the data packet the source station starts a countdown timer (i.e., timeout), which leaves enough time for the reception of the ACK frame. If the timeout (known as ACK_Timeout in the case of using the basic access method) expires before the correct reception of the ACK frame, then the source station assumes that the frame is lost and hence prepares to retransmit the data packet. When TCP traffic is employed, there is an additional handshake at the higher layer for the transmission of the TCP ACK packet back to the source station. This action provides confirmation of the successful reception of data at the TCP layer. Unlike a standard wireless system, where the fiber link is not present each packet in the transmission is associated with a fiber delay $(\mathrm{F})$ which needs to be taken into account when setting the ACK_Timeout. Otherwise, the timeout could be exceeded due to the fiber delay in which case the number of retransmissions increases and consequently the performance degrades significantly. This can even lead to a complete network shut-down [7].

In the case of using the optional request to send (RTS) and CTS access method, or four-way handshaking technique, short RTS/CTS frames are employed to ensure that the medium is clear before transmission. This means that the stations attempt to reserve the shared medium for the time duration needed to transfer the actual data frame prior to transmission. During this period, all stations in the reserved area are restricted from transmission even though the channel may be idle. The IEEE 802.11 standard adopts the RTS/CTS method when the size of the data frame is larger than a predefined threshold [3], [8] (2347 bytes within our experiment). Before transmitting a data frame, the source station transmits an RTS frame, and the destinations station replies with a CTS frame. After the transmission of the RTS frame the source station starts a countdown timer (CTS_Timeout), which leaves enough time for the reception of the CTS frame. If the expected response is not received within the CTS_Timeout, then the source station assumes that the frame is lost and hence prepares itself for retransmitting the RTS frame. Other stations in the vicinity hearing either an RTS or a CTS or both, defer their transmission by adjusting their network allocation vector (NAV), a timer, to the duration field value of the RTS/CTS frames.

\section{EXPERIMENTAL VALIDATION}

\section{A. Experimental Setup}

The experimental setup of a hybrid optical-wireless 802.11 broadband network is depicted in Fig. 3. The access point or base unit (BU) and remote bridge (RB) are each connected to an IEEE 802.3100 Base-T LAN. Using Ethernet limits the maximum MAC payload size to 1500 bytes (rather than 2312 bytes [9]-[11]). The uplink (where packets are transmitted from the $\mathrm{RB}$ to $\mathrm{BU}$ ) and downlink signals are separated using a RF circulator with $0.25 \mathrm{~dB}$ loss. A single fiber is used with different wavelength for uplink and downlink. To eliminate the effect of the wireless channel on the system performance an RF cable connects the RAU and the RB. The RBs are particular to the experimental setup being used, and their functionality in our experiment is simply to provide wireless access for the mobile units.

The uplink and downlink RF signals are separated using a circulator. The electrical signal is converted to an optical signal using a $2.5-\mathrm{GHz}$ bandwidth DFB laser. An optical combiner with 3.6-dB loss is used which allows a single length of SMF to be used for both directions. To ensure that the optical power levels in the system were constant, a bidirectional optical attenuator was employed for each fiber length. At the receiver end, an RF amplifier is used to increase the radio signal power level, which has a maximum value of $100 \mathrm{~mW}$ in Europe [3]. It should be noted that all amplification occurs at the RAU. 


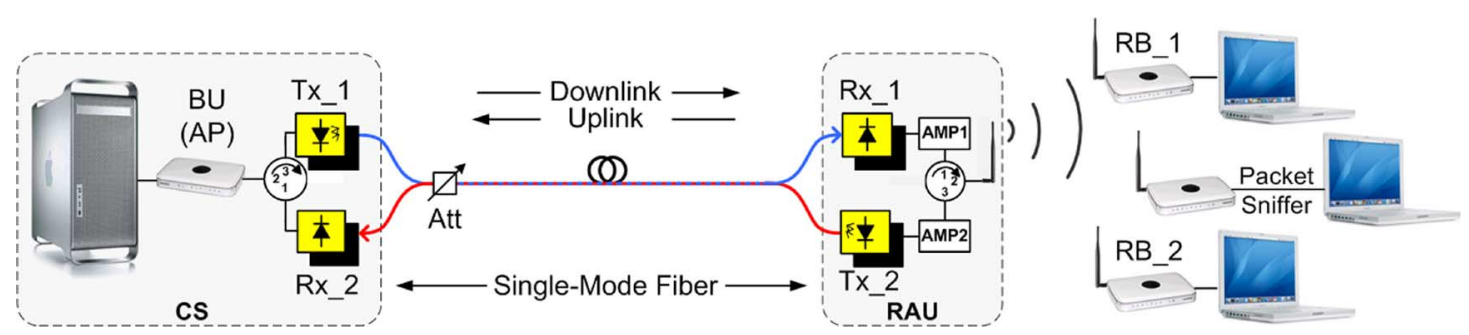

Fig. 3. Experimental Setup for short-range RoF systems.

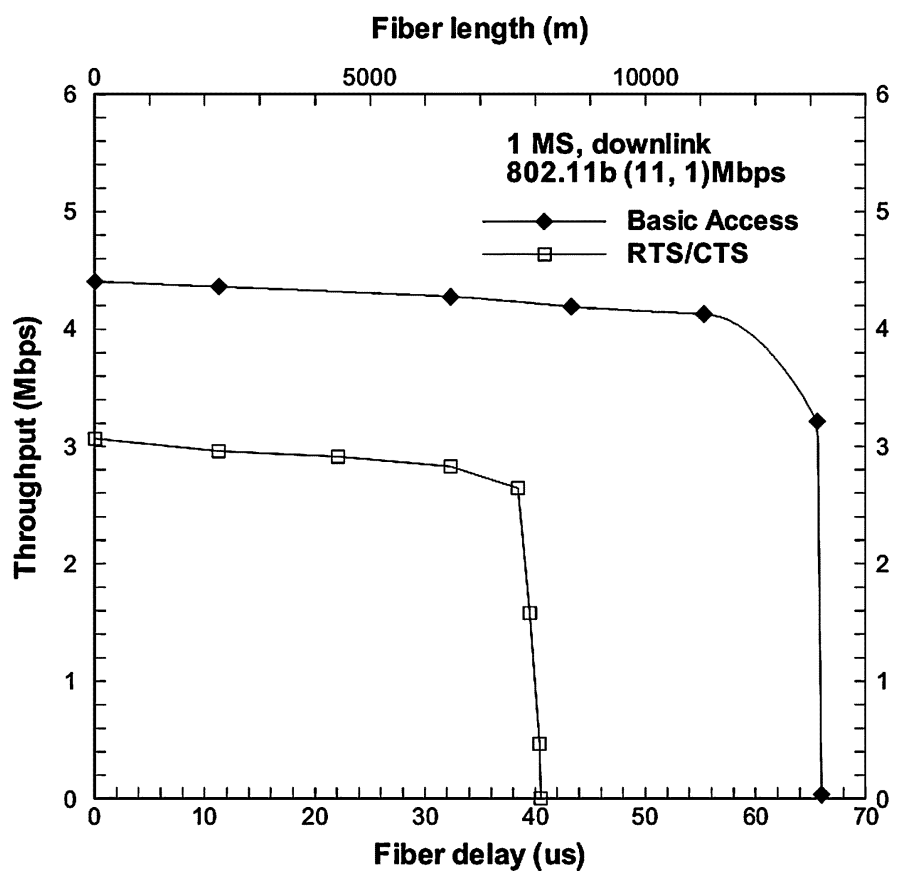

Fig. 4. Experimental result of an $11 \mathrm{Mbps}$ TCP_Basic and TCP_RTS $802.11 \mathrm{~b}$ system. Note, the ACK_Timeout is greater than the CTS_Timeout.

\section{B. Experimental Results}

There are two mobile nodes involved in the configuration shown in Fig. 3. This means that the station connected to RB_1 can receive packets from the station associated with $\mathrm{RB}_{-} 2$. The third node is used to capture (receive only mode) the packets being transmitted between the BU and RBs (or between the two $\mathrm{RBs}$ ). These packets are then processed by packet sniffing software. Fig. 4 shows the experimental downlink results of an 11 Mbps 802.11b system which transmits TCP traffic to one station via both basic access and RTS/CTS methods. Around 5\%-7\% of the data packets in the system are transmitted at a lower data rate of 5.5 Mbps (rather than $11 \mathrm{Mbps}$ ). It is common with all 802.11 systems that even before fiber is introduced the actual throughput is considerably lower than the given data rate due to the inherent overheads.

It can be observed from Fig. 4 that the throughput is decreased gradually from 4.48 Mbps (this is typical for non-RoF WLANs [12]) when no fiber is present to 4 Mbps over $11 \mathrm{~km}$ of fiber (an 11\% drop). As the system round-trip delay reaches the ACK_Timeout value the performance falls abruptly. The throughput sharply decreases when the ACK_Timeout expires. Since there is only one mobile station available at this stage
TABLE I

PARAMETERS USED WITHIN THE SYSTEM

\begin{tabular}{ll}
\hline Slot_Time ( $\sigma)$ & $20 \mu \mathrm{s}$ \\
SIFS (Short Inter-frame Space) & $10 \mu \mathrm{s}$ \\
DIFS =(2.Slot_Time+SIFS) & $50 \mu \mathrm{s}$ \\
PLCP Preamble \& Header & 24 bytes \\
MAC Header \& CRC & 34 bytes \\
Data rate & $11 \mathrm{Mbps}$ \\
Control rate & $1 \mathrm{Mbps}$ \\
RTS & 20 bytes \\
CTS or ACK & 14 bytes \\
Air propagation delay $(\delta)$ & $1 \mu \mathrm{s}$ \\
Fiber propagation delay $(F)$ & $1 \mu \mathrm{s}=194.8 \mathrm{~m}$ \\
$W$ (min Window size) & 32 \\
$m$ (max backoff stage) & 5 \\
$2^{m}$ W (max Window size) & 1024 \\
ACK_Timeout or CTS_Timeout & $(314+2 \delta) \mu s$ \\
\hline
\end{tabular}

the uplink and downlink performances are analogous. In the case of the RTS/CTS method, the maximum throughput is observed to be 3.1 Mbps (no fiber in the system) compared to 2.81 Mbps (with $6.5 \mathrm{~km}$ of fiber), i.e., a $9 \%$ reduction over $6.5 \mathrm{~km}$ of fiber. Since the ACK_Timeout $(450 \mu \mathrm{s})$ is greater than the CTS_Timeout value ( $399 \mu$ s) the cutoff in the throughput of the RTS/CTS method occurs earlier than the basic access method.

The timeout values are based on the minimum timeout value defined in the IEEE 802.11 standard, i.e., $314 \mu$ s $+\alpha$, where $\alpha$ varies depending on each vendor.

\section{AnAlytical ApPROXimation AND SimUlation SETUP}

This section briefly describes the simulation setup and introduces the analytical work to evaluate the performance of hybrid optical-wireless $802.11 \mathrm{~b}$ networks where TCP and UDP traffic are involved. We consider the basic access and RTS/CTS modes and investigate the effect that fiber delay (length) has on the resulting performance. We also take into account multiple stations, as well as, a single station, performing upload or download operations in a network of fiber lengths of up to $13.2 \mathrm{~km}$.

To model the hybrid optical-wireless $802.11 \mathrm{~b}$ broadband network operating in the infrastructure mode we have used OPNET Modeler 11.5. All uplink and downlink operations are carried out via the BU. The values of MAC payload size, data rate and control rate correspond to the values used in the experimental measurements and analytical work, see Table I. Whenever the MAC is considered in isolation from the higher layers, the Poisson process is used to model the arrival of the packets from the protocol layers above it.

Our analysis is based on the model presented by Bianchi in [13] which is purely for wireless networks. Here, we modify and extend the model to consider RoF systems. In addition, we also introduce terms to take into account the effects of ACK 


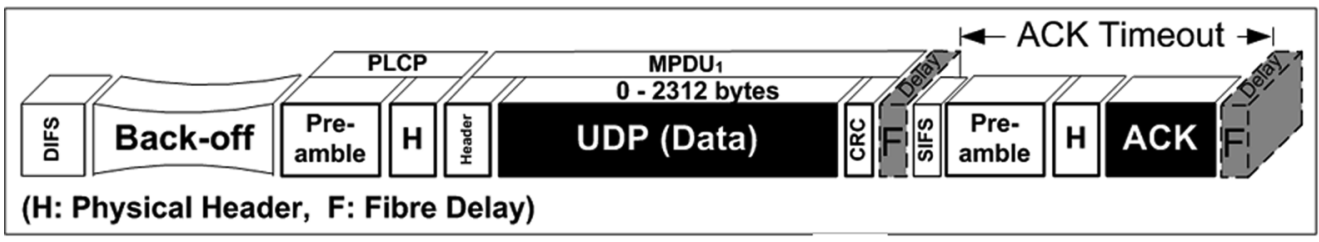

(a)

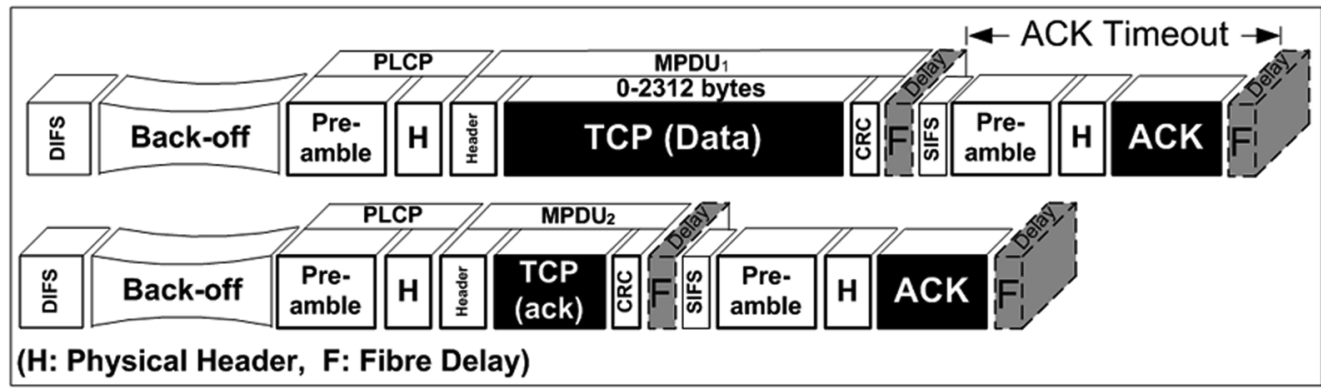

(b)

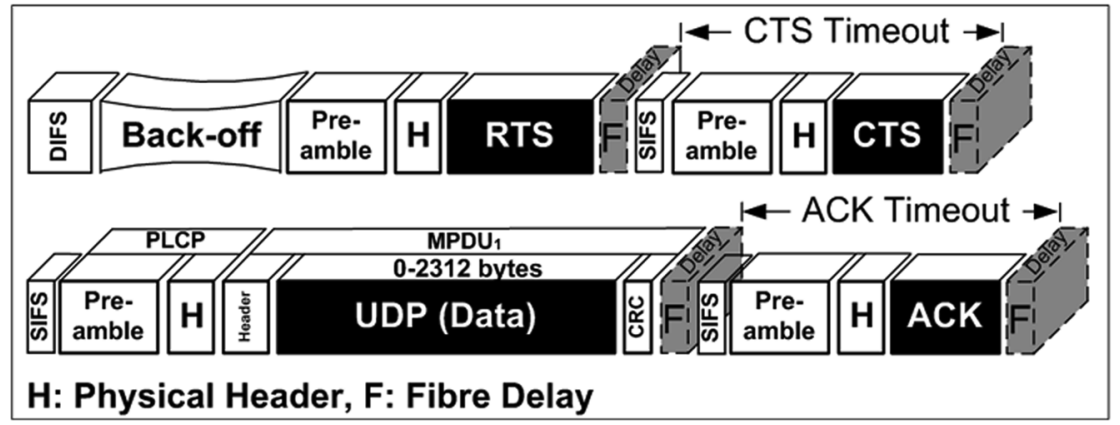

(c)

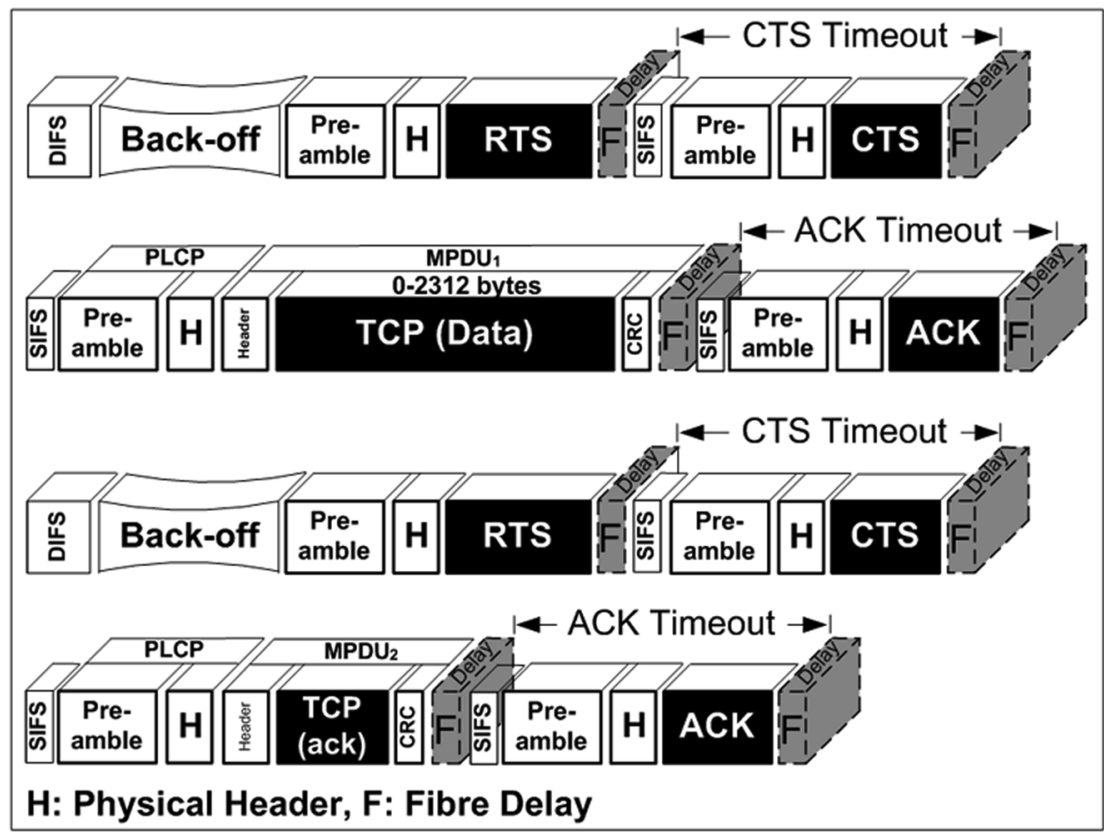

(d)

Fig. 5. Packet exchange of basic access and RTS/CTS methods when UDP and TCP traffic is used within a hybrid optical-wireless 802.11 network. (a) UDP traffic over basic access mechanism. (b) TCP traffic over basic access mechanism. (c) UDP traffic over RTS/CTS mechanism. (d) TCP traffic over RTS/CTS mechanism.

and CTS timeouts, as well as, TCP packet transmission over different lengths of fiber. For the purpose of clarity we use the same notation and equations used within [13] to model the backoff procedure of an 802.11 wireless network. 
Fiber length (m)

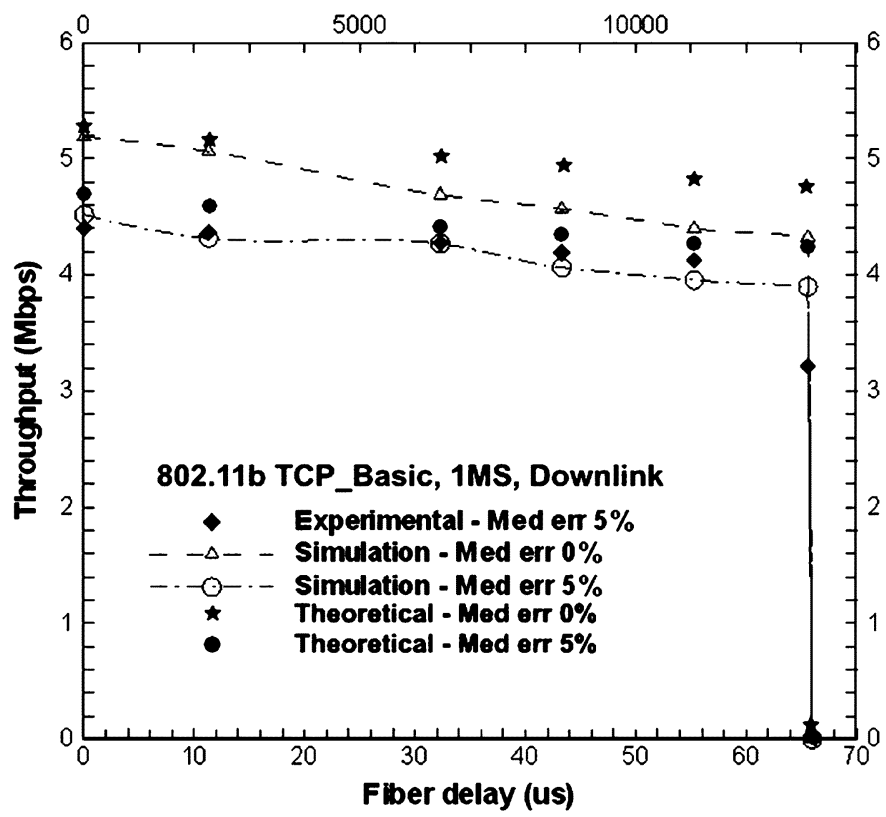

(a)

Fiber length (m)

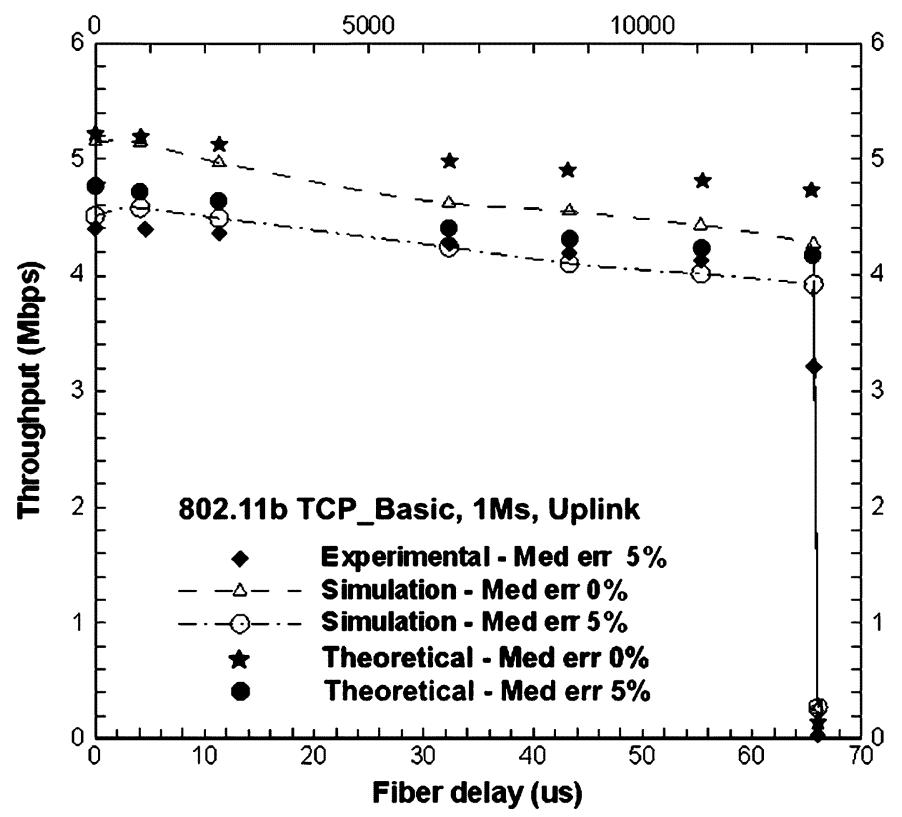

(b)

Fig. 6. Variation of downlink and uplink throughput with fiber length for TCP traffic when the basic access method is employed. (a) Downlink. (b) Uplink.

Assume that the network consists of $n$ contending stations which are saturated, i.e., they always have a packet ready for transmission. Table I defines the parameters used in our analysis.

Following the analysis in [13] we assume that the conditional collision probability $p$ (i.e., the probability that each packet collides) and the transmission probability $\tau$ (i.e., the probability that a station transmits in randomly chosen Slot_Time) are both constant and independent for each station. By knowing $p$ and $\tau$, the successful transmission probability in each slot can be obtained and the efficient channel utilization rate can be calculated. To get $p$ and $\tau$, a two dimensional Markov chain model
Fiber length (m)

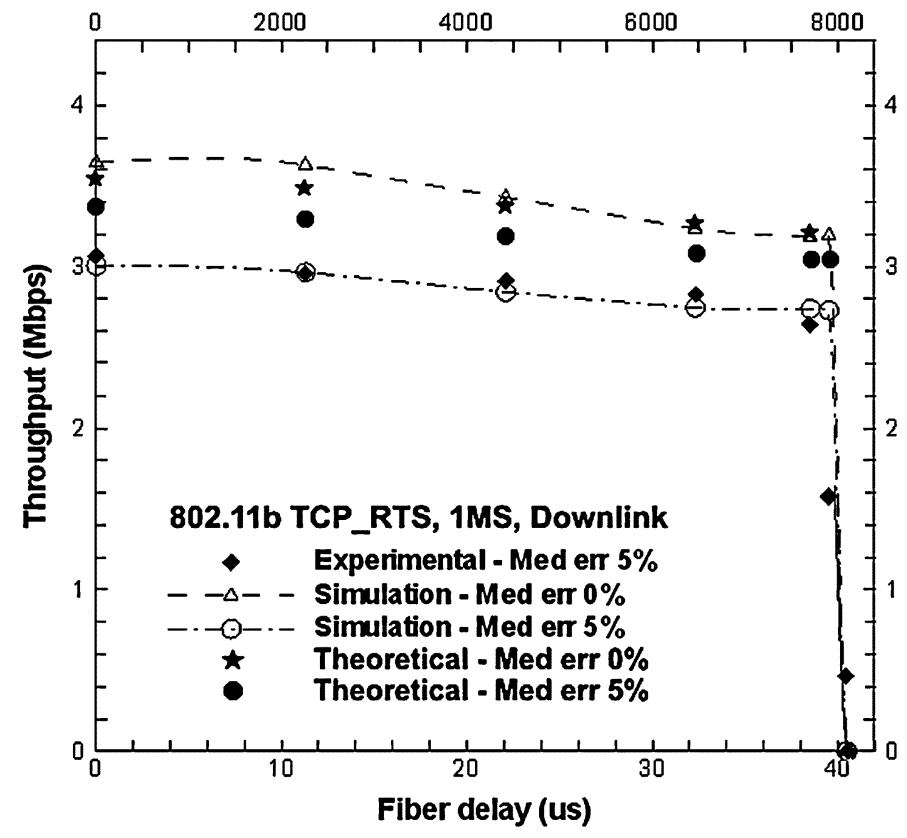

Fig. 7. Variation of downlink throughput with fiber length for TCP traffic when the RTS/CTS method is used. Uplink results are similar to downlink as there is only one mobile station (MS) contending the channel.

based on stationary probability $b_{i, k}$ is used to describe the saturation behavior of the 802.11 wireless network. Subsequently, by numerically solving the nonlinear system of (1), (2), and (3), the values of $p$ and $\tau$ can be obtained.

$$
\begin{aligned}
b_{0,0} & =\frac{2(1-2 p)(1-p)}{(1-2 p)(W+1)+p W\left(1-(2 p)^{m}\right)} \\
\tau & =\sum_{i=0}^{m} b_{i, 0}=\sum_{i=0}^{m} p^{i} \cdot b_{0,0} \\
& =b_{0,0} \cdot \frac{1-p^{m+1}}{1-p} \\
p & =1-(1-\tau)^{n-1} .
\end{aligned}
$$

To obtain the throughput we need to obtain $P_{\mathrm{tr}}$ (the probability of at least one transmission in each Slot_Time) and $P_{S}$ (the probability of a successful transmission in each Slot_Time) based on $\tau$ by (4) and (5).

$$
\begin{aligned}
P_{\mathrm{tr}} & =1-(1-\tau)^{n} \\
P_{S} & =\frac{n \tau \cdot(1-\tau)^{n-1}}{P_{\mathrm{tr}}}=\frac{n \tau \cdot(1-\tau)^{n-1}}{1-(1-\tau)^{n}} .
\end{aligned}
$$

Then the throughput can be calculated by using (6), shown at the bottom of the next page.

The average useful (i.e., data) packet payload size in bits is $E_{\text {[Packet] }}$. A successful transmission in a Slot_Time occurs with the probability of $P_{S} P_{\mathrm{tr}}$. The average time the wireless channel is sensed busy because of a successful transmission $T_{\text {Success }}^{\text {Operational_mode }}$ and because of an unsuccessful transmission $T_{\text {Failure }}^{\text {Operational }}$ mode depends on the operational mode, i.e., the mechanism and the type of packet traffic used by the system. 
If the basic access method is used over the fiber, according to Fig. 5, the following equations are obtained:

$$
\begin{aligned}
& T_{\text {Success }}^{\text {UDP_basic }} \\
& =\left\{\begin{array}{c}
\mathrm{DIFS}+2\left(T_{\mathrm{PLCP}}+F+\delta\right)+T_{\mathrm{ACK}}^{1 \mathrm{Mbps}}+\mathrm{SIFS} \\
+\left[P^{11 \mathrm{Mbps}} \cdot T_{\mathrm{MPDU}}^{11 \mathrm{Mbs}}+P^{5.5 \mathrm{Mbps}} \cdot T_{\mathrm{MPDU}}^{5.5 \mathrm{Mbps}}\right]
\end{array}\right. \\
& T_{\text {Failure }}^{\text {UDP_basic }} \\
& =\left\{\begin{array}{c}
\mathrm{DIFS}+T_{\mathrm{PLCP}}+\mathrm{ACK}_{\mathrm{L}} \text { Timeout }+2 F \\
\quad+\left[P^{11 \mathrm{Mbps}} \cdot T_{\mathrm{MPDU}_{1}}^{11 \mathrm{Mbps}}+P^{5.5 \mathrm{Mbps}} \cdot T_{\mathrm{MPDU}}^{5.5 \mathrm{Mbps}}\right]
\end{array}\right.
\end{aligned}
$$

$$
\begin{aligned}
& T_{\mathrm{Success}}^{\mathrm{TCP}} \\
& =\left\{\begin{array}{l}
2\left(T_{\mathrm{PLCP}}+F+\delta\right)+\mathrm{DIFS}+T_{\mathrm{ACK}}+\mathrm{SIFS} \\
+\left[P^{11 \mathrm{Mbps}} \cdot T_{\mathrm{MPDU}}^{11 \mathrm{Mbps}}+P^{5.5 \mathrm{Mbps}} \cdot T_{\mathrm{MPDU}_{1}}^{5.5 \mathrm{Mbps}}\right] \\
+P_{\mathrm{ack}}^{\mathrm{TCP}} \cdot\left\langle 2\left(T_{\mathrm{PLCP}}+F+\delta\right)+\mathrm{DIFS}+T_{\mathrm{ACK}}\right. \\
\left.+\mathrm{SIFS}+\left[P^{11 \mathrm{Mbps}} \cdot T_{\mathrm{MPDU}_{2}}^{11 \mathrm{Mbps}}+P^{5.5 \mathrm{Mbps}} \cdot T_{\mathrm{MPDU}_{2}}^{5.5 \mathrm{Mbps}}\right]\right\rangle
\end{array}\right.
\end{aligned}
$$

$$
\begin{aligned}
& T_{\text {Failure }}^{\mathrm{TCP} \_b a s i c} \\
& =T_{\text {Failure }}^{\mathrm{UDP}}
\end{aligned}
$$

where $T_{\mathrm{MPDU}_{1}}^{11 \mathrm{Mbps}}$ and $T_{\mathrm{MPDU}_{1}}^{5.5 \mathrm{Mbps}}$ are the period of time during which an entire MAC protocol data unit (MPDU) frame is transmitted at 11 and 5.5 Mbps, respectively. To increase the accuracy the same approximation can also be applied when a packet is sent at 2 or $1 \mathrm{Mbps}$. Here, as seen in the experiment, we consider transmission at 5.5 and $11 \mathrm{Mbps}$. The probability that a packet is sent with $11 \mathrm{Mbps}$ is $P^{11 \mathrm{Mbps}}$ and the probability for $5.5 \mathrm{Mbps}$ transmission is $P^{5.5 \mathrm{Mbps}}$, where $P^{11 \mathrm{Mbps}}+P^{5.5 \mathrm{Mbps}}=1$. Note that in Fig. 5 all packets are associated with a physical layer convergence protocol (PLCP) overhead, which consists of a header and preamble as they go through the physical layer (PHY) of the IEEE 802.11 network.

In the case of employing the RTS/CTS mode we use the following equations, which are again based on Fig. 5:

$$
\begin{aligned}
& T_{\text {Success }}^{\text {UDP_RTS }} \\
& \quad=\left\{\begin{array}{l}
\text { DIFS }+4\left(T_{\mathrm{PLCP}}+F+\delta\right) \\
+T_{\mathrm{RTS}}+T_{\mathrm{CTS}}+T_{\mathrm{ACK}}+3 \mathrm{SIFS} \\
+P^{11} \mathrm{Mbps} T_{\mathrm{MPDU}_{1}}^{11}+P^{5.5 \mathrm{Mbps}} T_{\mathrm{MPDU}_{1}}^{5.5 \mathrm{Mbps}}
\end{array}\right. \\
& T_{\text {Failure }}^{\mathrm{UDP} \_\mathrm{RTS}} \\
& =\text { DIFS }+T_{\mathrm{PLCP}}+T_{\mathrm{RTS}}+\mathrm{CTS} \text {-Timeout }+2 F \\
& T_{\text {Success }}^{\mathrm{TCP}}
\end{aligned}
$$

$$
=\left\{\begin{array}{c}
4\left(T_{\mathrm{PLCP}}+F+\delta\right)+\mathrm{DIFS}+T_{\mathrm{RTS}}+T_{\mathrm{CTS}} \\
\quad+T_{\mathrm{ACK}}+3 \mathrm{SIFS}+P^{11 \mathrm{Mbps}} \cdot T_{\mathrm{MPDU}_{1}}^{11 \mathrm{Mbps}} \\
\quad+P^{5.5 \mathrm{Mbps}} \cdot T_{\mathrm{MPDU}}^{5.5 \mathrm{Mbps}}+P_{\mathrm{ack}}^{\mathrm{TCP}}\langle\mathrm{DIFS} \\
+4\left(T_{\mathrm{PLCP}}+F+\delta\right)+T_{\mathrm{RTS}}+T_{\mathrm{CTS}}+T_{\mathrm{ACK}} \\
\left.+3 \mathrm{SIFS}+P^{11 \mathrm{Mbps}} T_{\mathrm{MPDU}_{2}}^{11 \mathrm{Mbps}}+P^{5.5 \mathrm{Mbps}^{5.5 \mathrm{Mbps}_{\mathrm{MPDU}}}}\right\rangle
\end{array}\right.
$$

TEP_RTS

$$
=T_{\text {Failure }}^{\text {UDP_RTS }} \text {. }
$$

where $P_{\text {ack }}^{\mathrm{TCP}}$ is the probability of a TCP acknowledgement packet transmission, which is dependent on the TCP protocol. For example, if each two TCP data packets are acknowledged by only one TCP ack frame, then $P_{\text {ack }}^{\mathrm{TCP}}=0.5$. For the case, where all TCP data frames are acknowledged at the TCP layer $P_{\text {ack }}^{\mathrm{TCP}}=1$. According to the packet sniffing software used within our experiment $P_{\text {ack }}^{\mathrm{TCP}}$ varies between 0.62 and 0.7 .

\section{RESULTS}

\section{A. Single Station TCP Transmission}

The experimental, analytical and simulation results of an 11-Mbps TCP_basic access system are plotted in Fig. 6. As expected, there is a gradual decrease in throughput as the fiber length increases. Since there is only one transmitting node, the uplink and downlink behaviors are very similar. The sudden fall in throughput witnessed is due to the ACK_Timeout expiration, which, as aforementioned, limits the maximum fiber length that can be inserted in such systems. The same conclusions apply for the uplink and downlink RTS/CTS transmission plotted in Fig. 7. Again, as expected, we see that with RTS/CTS throughput is inherently lower than with basic access. Channel errors affect the performance of the MAC protocol since they will increase the number of retransmissions at the MAC level.

Experimental measurements show that, due to this, 5\%-10\% of the packets are transmitted at 5.5 Mbps (and around $2 \%-5 \%$ at lower data rates). The simulation and theoretical results plotted in Fig. 6 and Fig. 7 show a steady decrease in throughput with the increase in the channel errors. As an example, for downloading operations using basic access, there is approximately a $12 \%$ drop in throughput when the media error increases by $5 \%$ regardless of fiber length. It is worth mentioning that the media error (which after packet corruption, forces the packets to travel at 5.5 Mbps) is uniformly distributed throughout the transmission time within the simulation. In this paper the media error (i.e., $P^{5.5}$ Mbps in our analysis) and frame error (FER) terms are used interchangeably. In addition, the MAC retry_threshold parameter which determines the number of retransmission attempts at the MAC level is chosen as 7, resulting in a robust error correction procedure at the MAC layer.

$$
\mathrm{Th}=\frac{P_{S} \cdot P_{\mathrm{tr}} \cdot E_{[\text {Packet }]}}{\left(1-P_{\mathrm{tr}}\right) \cdot \sigma+P_{S} \cdot P_{\mathrm{tr}} \cdot T_{\text {Success }}^{\text {Operational_mode }}+P_{C} \cdot P_{\mathrm{tr}} \cdot T_{\text {Failure }}^{\text {Operational_mode }}} .
$$


Therefore no retransmission at the TCP level was observed in our simulation.

\section{B. Unidirectional TCP Transmission to Multiple Stations}

The throughput decrease due to the fiber delay is again evident in the experimental, simulation and analytical results of Fig. 8(a) where two mobile stations (MS) using basic access are simultaneously transmitting upstream. The simulation results are shown for two different FER. In addition to the total throughput of the system, the experimental measurements of the individual throughputs of the two transmitting stations are also shown, indicating a fair distribution of the bandwidth.

The effect of the fiber delay is more significant in the two MSs case compared to the single MS case. This is further confirmed by the simulation and analytical results illustrated in Fig. 8(b) which compares the throughput performance of the uplink and downlink transmission for the single and 5-MSs case, where an increased slope for the uplink multistation case is observed. In the downlink transmission an increase in the number of stations does not affect the throughput. This can be easily understood by the fact that in the downlink it is only the AP sending data, so there is no risk of MPDU frames colliding with a MAC ACK.

The results for the RTS/CTS uplink transmission of two MSs shown in Fig. 8(c) indicate a lower performance compared to the basic access mode. In all the cases presented, the sudden drop in throughput occurs whenever the round trip delay, exceeds the ACK_Timeout or the CTS_Timeout intervals, for the basic access and RTS/CTS modes, respectively. We have observed a small ACK_Timeout difference in RB_1 and RB_2, (due to software version differences) which causes the throughput to drop at a slightly shorter fiber length for RB_1 when they operate individually. However, when the stations operate simultaneously, the sudden throughput drop occurs at the same fiber length for both MSs, which corresponds to the shorter ACK_Timeout setting, due to the drop in the data transmission rates caused by the multiple retransmissions of RB_1.

\section{UDP Transmission}

As an alternative transport protocol we have also evaluated UDP traffic for use in hybrid optical-wireless $802.11 \mathrm{~b}$ broadband networks. Fig. 9 illustrates simulation and analytical results for UDP throughput compared with TCP and the maximum achievable MAC throughput for downlink and uplink respectively when basic access method is used. These results show that the UDP throughput performance is comparable to that of the MAC itself, whereas the TCP throughput is lower, which is expected due to the TCP acknowledgement procedure overhead. Compared to the case with no fiber, the decrease in the UDP throughput is around 7\% when $13.1 \mathrm{~km}$ of fiber is used.

\section{DISCUSSION}

As presented in our analytical model, the designers of hybrid optical-wireless systems can choose a specific equation to evaluate and approximate the performance of their network. These equations cover four different optically distributed 802.11 systems: TCP_Basic, TCP_RTS, UDP_Basic and UDP_RTS. These equations, depending on the parameters used within the network, can be used to evaluate the minimum and maximum limits

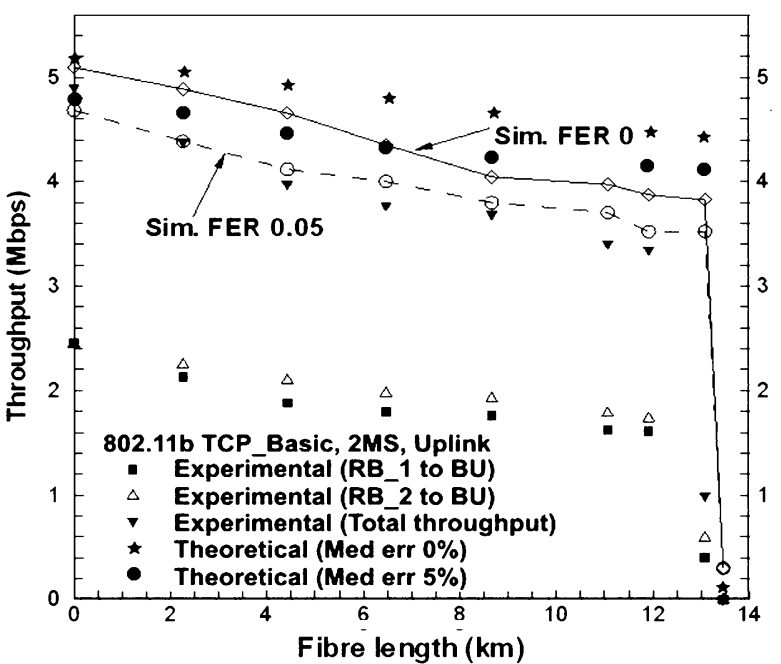

(a)

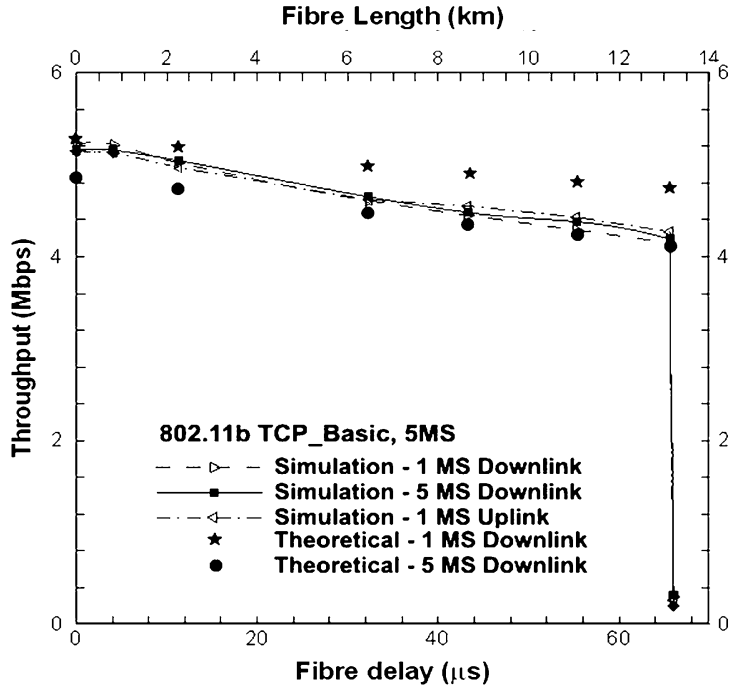

(b)

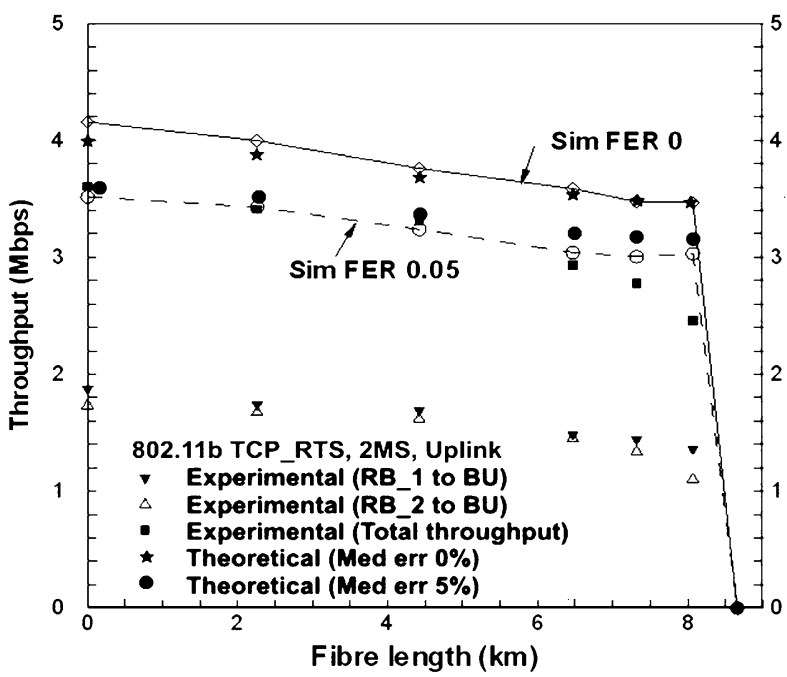

(c)

Fig. 8. Variation of throughput with fiber length for TCP traffic when basic access and RTS/CTS methods are used. (a) Basic access- uplink. (b) Basic access-uplink and downlink. (c) RTS/CTS.

that the system will operate within. For example, Fig. 10 shows the analytical results of an UDP_RTS system (with 2 nodes) that 


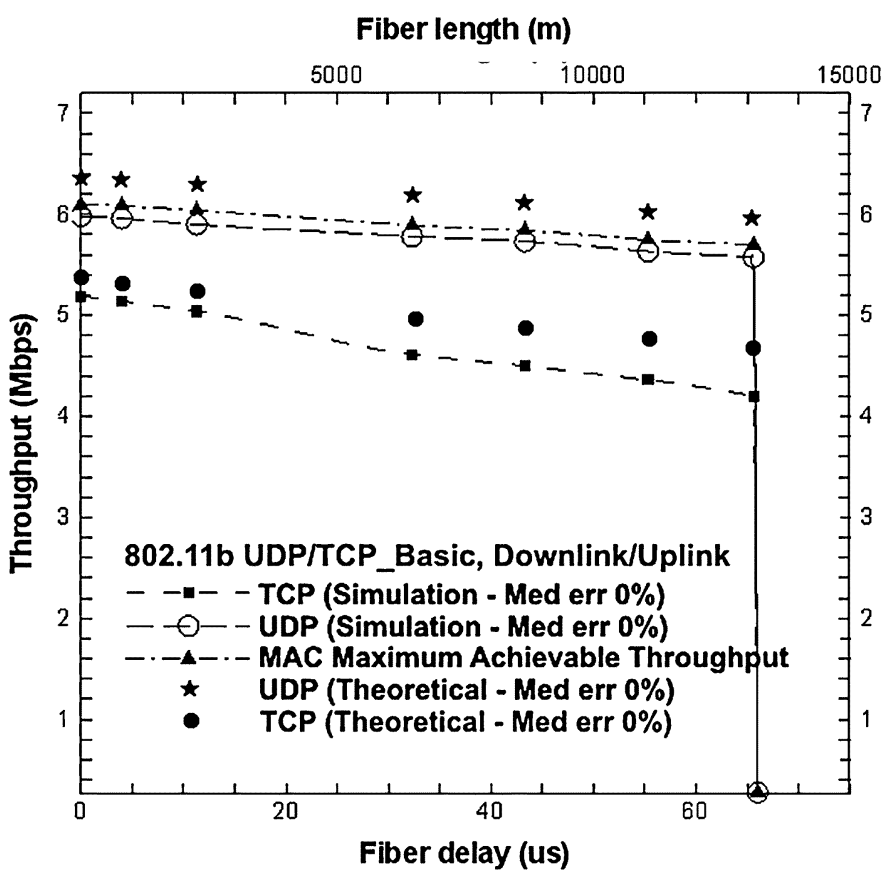

Fig. 9. UDP, TCP and the MAC maximum achievable throughput (downlink) when basic access method is used. Uplink results are very similar to downlink results as there is one MS contending the channel.

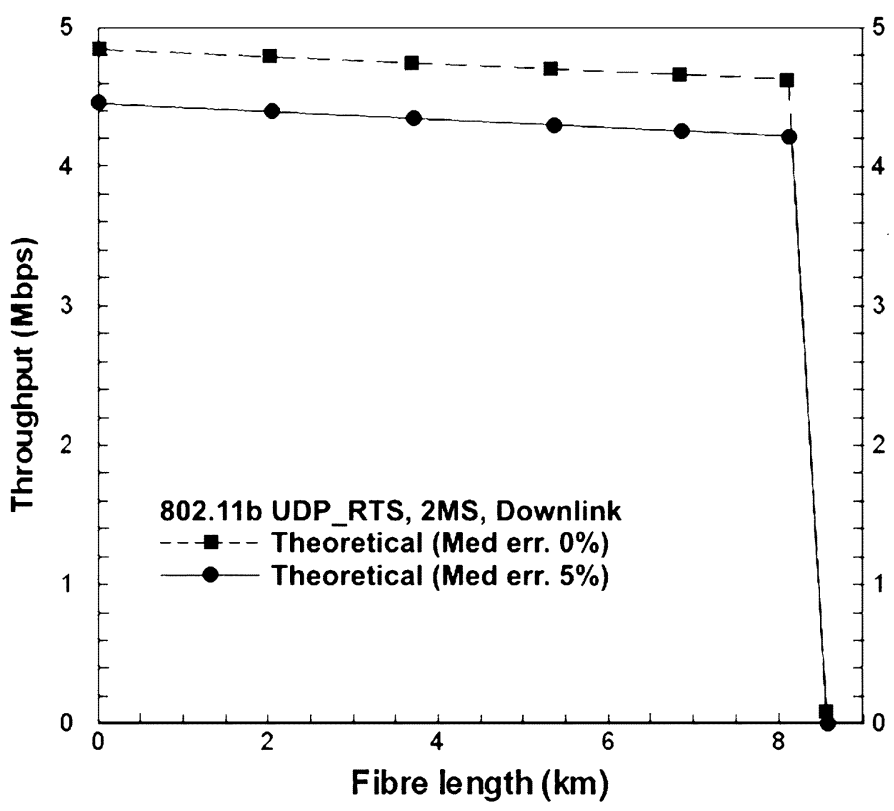

Fig. 10. Variation of throughput with fiber length for an UDP_RTS f.

has a maximum of 5\% media error $\left(P^{11}\right.$ Mbps $\left.=95 \%\right)$. In this case, the downlink throughput is around 5\% lower compared to a system with zero media error regardless of the length of the fiber. These could be considered as the upper and lower bounds on the expected operation.

\section{CONCLUSION}

In this paper, we have investigated the constraints and limitations faced on the performance of hybrid optical-wireless
802.11 broadband networks. Previous studies in this framework have indicated the behavior of such systems using short-range scenarios via multimode fiber. Most of these studies are carried out by simulations only. In this paper, we consider single-mode fiber and extend the system's performance analysis with an extensive set of measurements performed on a real testbed by employing commercially available IEEE $802.11 \mathrm{~b}$ cards. We consider the cases of a single mobile station, multiple mobile stations and bidirectional TCP and UDP traffic in the link. We demonstrate using experiment, simulations and theoretical approximation that there will be a small performance degradation due to the additional delay inherent in the fiber transmission, although this is typically less than $15 \%$. However, we also show that when the timeouts defined in the standard are exceeded a complete failure of the link results. Failure occurred after $13.2 \mathrm{~km}$ when the basic access method was used, and $8.1 \mathrm{~km}$ when RTS/CTS mechanism was employed. Finally, we present analytical approximations to our experimental and simulation results that allow designers of RoF systems to quickly and accurately predict the expected data throughput according to the specific parameters used within their networks.

\section{REFERENCES}

[1] A. Das, A. Nkansah, N. J. Gomes, I. J. Garcia, J. C. Batchelor, and D. Wake, "Design of low-cost multimode fiber-fed indoor wireless networks," IEEE Trans. Microw. Theory Tech., vol. 54, pp. 3426-3432, 2006.

[2] N. J. Gomes, A. Das, A. Nkansah, M. Mjeku, and D. Wake, "Multimode fiber-fed indoor wireless networks," in Int. Top. Meet. Microw. Photon. (MWP'06), 2006, pp. 1-4.

[3] IEEE Standard for Wireless LAN Medium Access Control (MAC) and Physical Layer (PHY) Specifications, ISO/IEC 8802-11:1999(E), 1999.

[4] M. V. Clark, K. K. Leung, B. McNair, and Z. Kostic, "Outdoor IEEE 802.11 cellular networks: Radio link performance," in IEEE Int. Conf. Commun. (ICC 2002), 2002, pp. 512-516.

[5] M. Mjeku, B. Kalantari-Sabet, J. E. Mitchell, and N. J. Gomes, "TCP and UDP performance over fibre-fed IEEE $802.11 \mathrm{~b}$ networks," presented at the 12th Microcoll Conf., Budapest, Hungary, 2006.

[6] B. Kalantari-Sabet and J. E. Mitchell, "Identifying the MAC limitations of the optically distributed 802.11b network," in ISIS Summer School Workshop, Boppard, Germany, May 2006.

[7] B. Kalantari-Sabet and J. E. Mitchell, "MAC constraints on the distribution of 802.11 using optical fibre," in 9th Europ. Conf. Wireless Technol., 2006, pp. 238-240.

[8] Y. Shiang-Rung, W. You-Chiun, and T. Yu-Chee, "A jamming-based MAC protocol for wireless multihop ad hoc networks," in IEEE 58th Veh. Technol. Conf. VTC 2003-Fall, 2003, vol. 3, pp. 1396-1400.

[9] IEEE Std. 802.11a-1999, Part 11: Wireless LAN Medium Access Control (MAC) and Physical Layer Specifications: High Speed Physical Layer in the $5 \mathrm{GHz}$ Band, , 1999.

[10] Part 11: Wireless LAN Medium Access Control (MAC) and Physical Layer (PHY) Specifications: Higher-Speed Physical Layer Extension in the $2.4 \mathrm{GHz}$ Band, IEEE Std 802.11b-1999, 1999.

[11] Part 11: Wireless LAN Medium Access Control (MAC) and Physical Layer (PHY) Specifications: Further Higher Data Rate Extension in the 2.4 GHz Band, IEEE Std 802.11g-2003, 2003.

[12] G. Anastasi, E. Borgia, M. Conti, and E. Gregori, "IEEE 802.11b ad hoc networks: Performance measurements," Cluster Computing, vol. 8, pp. 135-145, 2005.

[13] G. Bianchi, "Performance analysis of the IEEE 802.11 distributed coordination function," IEEE J. Sel. Areas Commun., vol. 18, pp. 535-547, 2000 . 


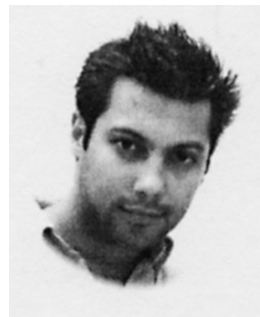

Bahman Kalantari-Sabet received the B.Eng. (with First-Class Honors) degree in electronic and electrical engineering from King's College London (KCL), University of London, London, U.K., in 2004.

He is currently working toward the Ph.D. degree at Telecommunication Research Group, Department of Electronic and Electrical Engineering, University College London (UCL). His research interests include radio over fiber communication and network systems with particular attention to MAC protocols in hybrid optical-wireless 802.11 broadband networks.

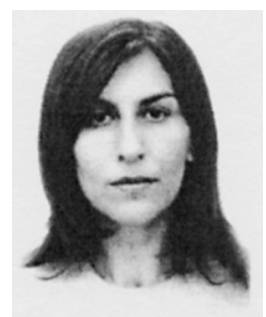

Majlinda Mjeku received the B.Eng. (Hons.) degree in electronic-telecommunication engineering from the Polytechnic University of Tirana, Tirana, Albania, in 1996 and the M.Sc. degree in object oriented software systems from City University London, U.K., in 2002.

She is currently working toward the Ph.D. degree in electronic engineering at Broadband and Wireless Communications Group, Department of Electronics, University of Kent, Canterbury, U.K. Her current research interests include protocol effects in

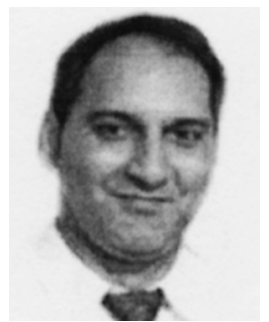

Nathan J. Gomes (M'92-SM'06) received the B.Sc. degree from the University of Sussex, U.K., in 1984 and the Ph.D. degree from University College London, U.K., in 1988, both in electronic engineering.

From 1988 to 1989 , he held a Royal Society European Exchange Fellowship at ENST, Paris. Since late 1989, he has been a Lecturer, and since 1999, a Senior Lecturer, in the Electronics Department, University of Kent, U.K. His present research interests include radio over fiber systems and networks, the photonic generation and transport of millimeter-wave signals, and photoreceivers for such applications.

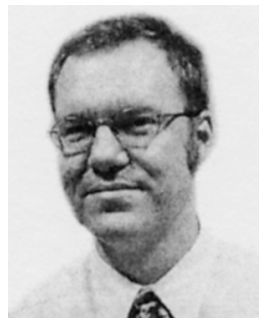

John E. Mitchell (S'97-M'00) received the B.Eng. degree in electronic and electrical engineering and the Ph.D. degree from University College London (UCL), U.K., in 1996 and 2000, respectively.

In 2000, he was appointed as a Lecturer with the Department of Electronic and Electrical Engineering, UCL, becoming a Senior Lecturer in 2006. He has published more than 40 papers in the area of optical communications. His research interests include optical access technologies (in particular, long reach and WDM-PONs) and the transport of millimeter-wave radio signals over optical fiber for communications and noise relating to crosstalk in optical networks.

Dr. Mitchell has presented invited talks and tutorials at a number of conferences, workshops, and meetings, both nationally and internationally, on the subject of radio-over-fiber networks and optical access. He is a member of the Institution of Engineering and Technology and the IEEE Lasers and Electro-Optics Society. 